\title{
All in for nuclear PFKP-induced CXCR4 metastasis: a T cell acute lymphoblastic leukemia prognostic marker
}

\author{
Hal E. Broxmeyer \\ Department of Microbiology and Immunology, Indiana University School of Medicine, Indianapolis, Indiana, USA.
}

\begin{abstract}
Phosphofructokinase 1 (PFK1) is expressed in T cell acute lymphoblastic leukemia (T-ALL), where its upregulation is linked with cancer progression. While PFK1 functions in the glycolysis pathway within the cytoplasm, it is also present in the nucleus where it regulates gene transcription. In this issue of the $J C l$, Xueliang Gao, Shenghui Qin, et al. focus their mechanismbased investigation on the nucleocytoplasmic shuttling aspect of the PFK1 platelet isoform, PFKP. Functional nuclear export and localization sequences stimulated CXC chemokine receptor type 4 (CXCR4) expression to promote T-ALL invasion that involved cyclin D3/CDK6, c-Myc, and importin-9. Since the presence of nuclear PFKP is associated with poor survival in T-ALL, nuclear PFKP-induced CXCR4 expression might serve as a prognostic marker for T-ALL. More promising, though, are the mechanistic insights suggesting that approaches to dampening metastatic migration may have application to benefit patients with T-ALL.
\end{abstract}

signals. Notably, the nuclear localization signal was displayed at the protein surface in the dimeric form, but not the tetrameric form. Furthermore, higher quantities of PFKP dimer were observed in the nucleus. The researchers were able to reduce levels of nuclear PFKP by depleting leukemia cells of cyclin D3 and CDK6. Biochemical assays showed a direct interaction between cyclin D3/CDK6 and a PFKP binding motif. The authors went on to show that inhibiting CDK6 decreased leukemia cell invasion. Importantly, inhibiting CDK6 also decreased CXCR4 expression. Conversely, when nuclear PFKP was increased, CXCR4 expression also increased (15). T-ALL CXCR4 expression driven by nuclear PFKP distinguishes this present work (15) from prior studies involving CXCR4 expression and migration/metastasis of T-ALL or CXCR4 function in normal and malignant cell biology (Figure 1).

\section{CXCR4 expression via nuclear PFKP in T-ALL}

Phosphofructokinase 1 (PFK1) is an important $340 \mathrm{kDa}$ regulatory allosteric enzyme of glycolysis composed of four subunits that are controlled by many activators and inhibitors. There are three different PFK1 isoforms, muscle, liver, and platelet, which differ depending on the tissue of residence. Although PFK1 is found in the cytoplasm, recent data suggest that it can also translocate to the nucleus to regulate transcription. However, the genes that PFK1 may regulate remained unknown.

The CXC chemokine receptor type 4 (CXCR4), which is found on the cell surface and binds the chemokine stromal cell-derived factor 1 (SDF-1, also known as CXCL12), is well known for its intimate and crucial involvement in chemotaxis (directed cell movement), migration, homing, and mobilization of normal hematopoietic stem cells (HSCs), hematopoietic progenitor cells (HPCs), and more mature lymphoid cells (1-7). CXCR4 has also been implicated in the movement and metastasis of leukemia and cancer cells and leukemia/cancer stem/initiating cells (8-14).

In this issue of the JCI, Xueliang Gao, Shenghui Qin, et al. focused on the platelet isoform of PFK1 (PFKP) and CXCR4 (15). The authors provide mechanistic insights with potential diagnostic and perhaps clinical translational possibilities to ameliorate the metastatic migration potential of T-ALL cells. Previously, these investigators established that PFKP is the major isoform of PFK1 that is expressed in T-ALL (16). Xueliang Gao, Shenghui Qin, et al. now evaluate CXCR4-dependent T-ALL infiltration mediated by nuclear PFKP. The authors compared results from mutational analysis of the known crystal structure to study potential nuclear localization and export
Related Article: https://doi.org/10.1172/JCl143119

Conflict of interest: HEB is a member of the Scientific Advisory Board of Eixell, a cord blood hematopoietic stem cell expansion company, and receives a consultant fee for his services.

Copyright: @ 2021, American Society for Clinical Investigation.

Reference information: / Clin Invest. 2021;131(16):e151295. https://doi.org/10.1172/JCl151295.

\section{Remaining questions and suggested possible future efforts}

Xueliang Gao, Shenghui Qin, et al. (15) bring up a number of interesting possibilof T-ALL migration/metastasis (Figure 1). Some questions to put forward include: What is the role of the CXCL12/CXCR4 axis in enhancing survival? How does this axis affect T-ALL cells? What is its involvement with enhanced migration/ metastasis of T-ALL cells, and perhaps other leukemias/cancers and leukemia/ cancer stem/initiating cells? Does the other CXCL12 receptor, CXCR7, play any role in T-ALL or other leukemias/cancers? What other receptors and their ligands (e.g., integrins) play a role in migration/ metastasis of T-ALL cells? Does nuclear localization of PFKP influence expression of other intracellular or cell surface molecules involved in migration/metastasis? What is the role of lowered oxygen tension in these processes in vivo in cells exposed to ambient air (approximately ities for possible future efforts in the area 


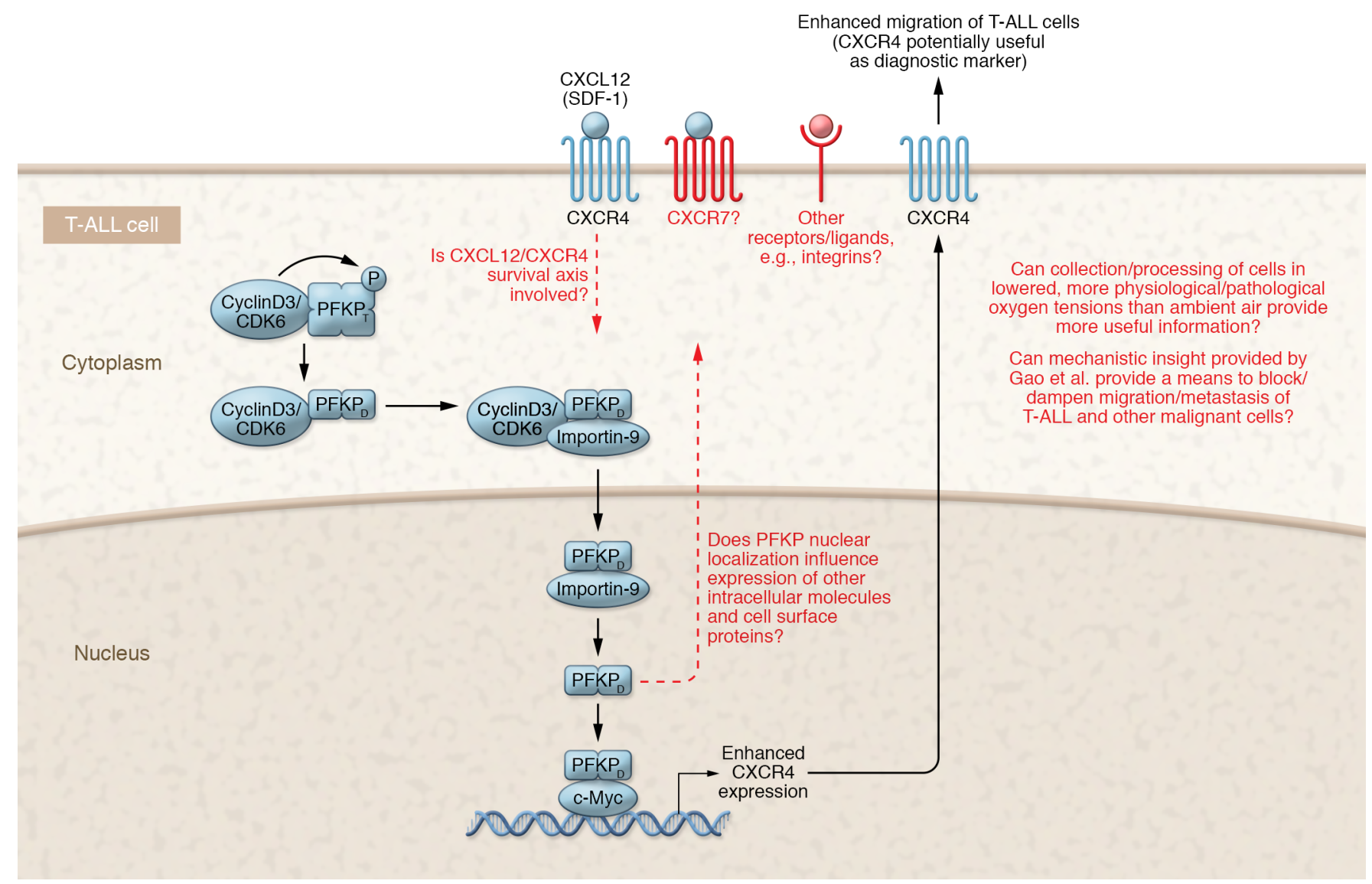

Figure 1. Diagrammatic representation of nuclear PFKP-induced CXCR4 in T-ALL. Gao, Qin, et al. showed that PNKP shuttles to the nucleus to enhance CXCR4 expression on T-ALL cells, which in turn enhances T-ALL migration into the bone marrow, spleen, and liver (15). These results bring up a number of interesting possibilities and future research questions.

$21 \%$ oxygen) upon removal, which is not physiologic/pathologic? Of equal or greater importance to the interesting potential diagnostic marker possibilities noted by the authors is whether the mechanistic insight of Xueliang Gao, Shenghui Qin, et al. (15) can provide a means to slow or block the metastatic process of T-ALL and/or other leukemias/cancers.

The CXCL12/CXCR4 axis has been implicated in survival of HSC/HPC populations $(4,17,18)$. A worthwhile endeavor would be to determine how such survival mechanisms fit into the schema provided by Xueliang Gao, Shenghui Qin, et al. (15). Although CXCL12 has another receptor, CXCR7 (19), this receptor is mainly relegated to the list of recently non-evaluated CXCL12 receptors. Is CXCR7 expressed on these T-ALL cells, and does nuclear localization of PFKP play any role in its expression and the migration/metastatic properties of T-ALL and other cells? There are numerous cell surface molecules involved in migration, e.g., integrins. Are these other surface molecules involved in the migration and metastases of T-ALL cells, as noted by Xueliang Gao, Shenghui Qin, et al. (15), and if so, how? CXCR4 is a hypoxia-regulated molecule (20). How might hypoxia in vivo and in vitro influence T-ALL cell migration? This hypoxia-related question is nuanced, as cell culture and cell processing are influenced by in vitro hypoxia (21). Further, whether cells are harvested under hypoxic or normoxic (e.g., ambient air and in vivo oxygen) conditions can lead to greatly different results, with hypoxic collection in addition to hypoxic processing being more physiologically, and possibly pathologically, relevant, at least for HSCs. Collection and processing of human cord blood, mouse bone marrow (22), and mobilized peripheral blood (23) allows detection of greatly increased numbers of normal HSCs and bone marrow cells from mice with Fanconi's anemia (24) compared with that collected/processed in ambient air.

\section{Dampening the metastatic potential of leukemia/cancer cells}

Although the potential to use CXCR4 as a diagnostic marker for T-ALL (15) is important, what we all hope for in the future is a means to harness the study's mechanistic insights to perhaps dampen the metastatic potential of the T-ALL and other leukemia cells while disrupting cancer/leukemia initiating/stem cell populations. The authors (15) used CXCL12/CXCR4 antagonists such as plerixafor/AMD310 on T-ALL cells with upregulated CXCR4 expression and showed that the cells' migratory processes were enhanced by nuclear localization of PFKP and associated interactions. Although such antagonists of CXCL12/ CXCR4 have been used to enhance basic knowledge and improve HSC/HPC proliferation, survival, and mobilization $(4,24)$, it will clearly take more insight to develop clinically relevant approaches to disrupt migration and metastases in T-ALL and 
other hematologic malignancies, which are notable future efforts.

We look forward with interest to a time in the near future when migration and metastasis of leukemia/cancer cells and their stem/initiating cell populations can be abrogated or dampened to improve patient outcomes.

\section{Acknowledgments}

Some of HEB's publications cited in this Commentary were supported by US Public Health Service Grants from the NIH: R35 HL139599 (Outstanding Investigator Award) and U54 DK106846 Cooperative Centers of Excellence in Hematology (CCEH).

Address correspondence to: Hal E. Broxmeyer, Department of Microbiology/ Immunology, Indiana University School of Medicine, 950 West Walnut Street, Bldg. R2, Room 302, Indianapolis, Indiana 46202-5121, USA. Phone: 317.274.7510; Email:hbroxmey@iupui.edu.

\footnotetext{
1. Broxmeyer HE, Capitano ML. Cytokines/ chemokines/other growth regulators and their receptors. In: Hoffman R, et al, ed. Hematology: Basic Principles and Practice. 8th ed. 2022. In press.

2. $\mathrm{Kim} \mathrm{CH}$, Broxmeyer HE. In vitro behavior of hematopoietic progenitor cells under the influence of chemoattractants: stromal cell-derived factor-1, steel factor, and the bone marrow environment. Blood.1998;91(1):100-110.

3. Peled A, et al. Dependence of human stem cell
}

engraftment and repopulation of NOD/SCID mice on CXCR4. Science. 1999;283(5403):845-848.

4. Capitano ML, et al. CXCL12/stromal cell derived factor-1 and hematopoiesis. In: Encyclopedia of Cell Biology. 2nd ed. 2021. In press.

5. Guo B, et al. Glucocorticoid hormone-induced chromatin remodeling enhances human hematopoietic stem cell homing and engraftment. Nat Med. 2017;23(4):424-428.

6. Xu D, et al. Pharmacological activation of nitric oxide signaling promotes human hematopoietic stem cell homing and engraftment. Leukemia. 2021;35(1):229-234.

7. Huang $X$, et al. Neutralizing negative epigenetic regulation by HDAC5 enhances human haematopoietic stem cell homing and engraftment. Nat Commun. 2018;9(1):2741.

8. Mehta SA, et al. Negative regulation of chemokine receptor CXCR4 by tumor suppressor p53 in breast cancer cells: implications of p53 mutation or isoform expression on breast cancer cell invasion. Oncogene. 2007;26(23):3329-3337.

9. Shi Y, et al. The role of the CXCL12/CXCR4/ CXCR7 chemokine axis in cancer. Front Pharmacol. 2020;11:574667.

10. Tulotta $\mathrm{C}$, et al. CXCR4 signaling regulates metastatic onset by controlling neutrophil motility and response to malignant cells. Sci Rep. 2019;9(1):2399.

11. Mukherjee D, Zhao J. The role of chemokine receptor CXCR4 in breast cancer metastasis. Am J Cancer Res. 2013;3(1):46-57.

12. Zhu Q, et al. High CXCR4 expression predicts a poor prognosis in resected lung adenosquamous carcinoma. J Cancer. 2020;11(4):810-818.

13. Karagiannis GS, et al. Emerging roles of Cxcl12/ Cxcr4 signaling axis in breast cancer metastasis. J Immunol. 2020;204(1 supplement):90.4

14. Masuda T, et al. Nuclear expression of chemokine receptor CXCR4 indicates poorer prognosis in gastric cancer. Anticancer Res. 2014;34(11):6397-6403.
15. Gao X, et al. Nuclear PFKP promotes CXCR4-dependent infiltration by T cell acute lymphoblastic leukemia. JClin Invest. 2021;131(6):e143119.

16. Wang $\mathrm{H}$, et al. The metabolic function of cyclin D3-CDK6 kinase in cancer cell survival. Nature. 2017;546(7658):426-430.

17. Broxmeyer HE, et al. Transgenic expression of stromal cell-derived factor-1/CXC chemokine ligand 12 enhances myeloid progenitor cell survival/antiapoptosis in vitro in response to growth factor withdrawal and enhances myelopoiesis in vivo. J Immunol. 2003;170(1):421-429.

18. Broxmeyer HE, et al. Stromal cell-derived factor-1/CXCL12 directly enhances survival/antiapoptosis of myeloid progenitor cells through CXCR4 and G(alpha)i proteins and enhances engraftment of competitive, repopulating stem cells. J Leukoc Biol. 2003;73(5):630-638.

19. Altenburg JD, et al. A naturally occurring splice variant of CXCL12/stromal cellderived factor 1 is a potent human immunodeficiency virus type 1 inhibitor with weak chemotaxis and cell survival activities. J Virol. 2007;81(15):8140-8148.

20. Schioppa T, et al. Regulation of the chemokine receptor CXCR4 by hypoxia. J Exp Med. 2003;198(9):1391-1402.

21. Huang $X$, et al. Hypoxia signaling pathway in stem cell regulation: good and evil. Curr Stem Cell Rep. 2018;4(2):149-157.

22. Mantel CR, et al. Enhancing hematopoietic stem cell transplantation efficacy by mitigating oxygen shock. Cell. 2015;161(7):1553-1565.

23. Aljoufi A, et al. Collection and processing of mobilized mouse peripheral blood at lowered oxygen tension yields enhanced numbers of hematopoietic stem cells. Stem Cell Rev Rep. 2020;16(5):946-953.

24. Broxmeyer HE, et al. Rapid mobilization of murine and human hematopoietic stem and progenitor cells with AMD3100, a CXCR4 antagonist. J Exp Med. 2005;201(8):1307-1318. 